\title{
Chromatin modifications associated with diabetes
}

Short title; chromatin, epigenetics and diabetes

Samuel T. Keating (1) and Assam El-Osta(1,2,3,4)

Corresponding author: assam.el-osta@bakeridi.edu.au

Telephone 613-8532-1389

Fax 613-8532-1100

Word count: 5959

1) Epigenetics in Human Health and Disease Laboratory, 2) Epigenomics Profiling Facility, Baker IDI Heart and Diabetes Institute, The Alfred Medical Research and Education Precinct, 16 Melbourne, Victoria 3004, Australia;

17 3) Department of Pathology, The University of Melbourne, Victoria 3010, Australia;

4) Faculty of Medicine, Monash University, Victoria 3800, Australia 


\section{Abstract}

21 Accelerated rates of vascular complications are associated with diabetes mellitus.

22 Environmental factors including hyperglycemia contribute to the progression of diabetic

23 complications. Epidemiological and experimental animal studies identified poor glycemic

24 control as a major contributor to the development of complications. These studies suggest

25 that early exposure to hyperglycemia can instigate the development of complications that present later in the progression of the disease, despite improved glycemic control. Recent experiments reveal a striking commonality associated with gene-activating hyperglycemic events and chromatin modification. The best characterized to date are associated with the chemical

29 changes of amino-terminal tails of histone H3. Enzymes that write specified histone tail 30 modifications are not well understood in models of hyperglycemia and metabolic memory as well

31 as human diabetes. The best-characterized enzyme is the lysine specific Set7 methyltransferase.

32 The contribution of Set7 to the etiology of diabetic complications may extend to other 33 transcriptional events through methylation of non-histone substrates. 


\section{Introduction}

38 Diabetes mellitus is characterised by chronic hyperglycemia induced by loss of insulin producing pancreatic $\beta$-cells (Type I diabetes) or progressive loss of insulin sensitivity and $\beta$ cell dysfunction (Type II diabetes). It is estimated there are more than 1.7 million sufferers in Australia [1] with worldwide estimates suggesting 150 million patients as of 2003. This is expected to increase to 300 million individuals with the disease by 2025 [2]. Both forms of the disease are associated with accelerated rates of microvascular complications including retinopathy, neuropathy, and nephropathy as well as macrovascular complications such as hypertension, atherosclerosis and stroke. Hyperglycemia presents as a major risk factor for the development of diabetic complications, particularly endothelial dysfunction associated with vascular complications [3]. The vascular damage arising from hyperglycemic insult acts upstream of the overproduction of reactive oxygen species (ROS) by the mitochondrial electron transport chain [4]. Importantly this has been demonstrated to activate the $\mathrm{NF}_{\kappa} \mathrm{B}$

Several large-scale studies highlight the clinical benefits of strict glycemic control and revealed that early hyperglycemic events instigate the development of diabetic complications that present much later in the progression of the disease $[5,6]$. In conjunction with studies in experimental animal models and cell culture, these observations imply that a cell may retain a memory of past hyperglycemic events that culminate in altered and persistent gene expression that drives the disease phenotype. This concept has been termed 'metabolic memory' or the 'legacy effect' [5,6]. Current knowledge suggests that chromatin modifications and epigenetic determinants play a key role in the establishment and progression of metabolic memory and persistent gene expression.

In this review we highlight the epidemiological evidence and experimental studies that demonstrate the detrimental effects of hyperglycemia and support the concept of metabolic memory with a particular focus on Type I diabetes. Furthermore we discuss the current 65 knowledge surrounding epigenetic mechanisms that link hyperglycemia-induced vascular 66 injury to chromatin modifications and altered gene expression as well as metabolic memory. 67 We discuss emerging experimental evidence of changes in genomic methylation and 68 posttranslational modifications such as acetylation and methylation of histone tails, before 69 focusing on perhaps the best characterized methyl-writing enzyme involved in lysine mono- 
methylation. Additionally we review recent advances in the understanding of Set7-mediated molecular mechanisms that could drive altered gene expression in the diabetic setting.

\section{Epidemiological studies}

Large-scale studies have highlighted the requirement for strict glycemic control in delaying the onset of diabetic vascular complications. The Diabetes Control and Complications Trial (DCCT) was conducted with a cohort of 1441 Type I diabetic individuals and a mean follow-up of 6.5 years with the aim to compare conventional and intensive glycemic control regimens [7]. Original findings of the DCCT reported that intensive glycemic control reduced the onset and progression of long-term diabetic complications that included neuropathy, nephropathy and retinopathy compared with patients that received conventional therapy [5]. At the conclusion of this trial, a follow-up observational study, the Epidemiology of Diabetes Intervention and Complications (EDIC) examined the long-term effects of the original DCCT cohort. While the DCCT concluded that the development of cardiovascular complications were indistinguishable across intensive and conventionally treated groups, the EDIC trial identified a significantly lower risk of retinal and renal disease in the group that received intensive treatment [8]. At the conclusion of the DCCT, glycosylated hemoglobin $\left(\mathrm{HbA}_{1 \mathrm{c}}\right)$ levels differed by approximately $2 \%$ between the two groups [5]. Intriguingly towards the end of the EDIC study, $\mathrm{HbA}_{1 c}$ levels of both groups had converged to comparable levels [8]. Despite the normalization of $\mathrm{HbA}_{1}$, the effects of intensive therapy conducted for 6.5 years during the DCCT were persistently beneficial for at least 10 years with regard to microvascular complications. More recently it was reported that patients that received continuous intensive treatment throughout the trials were at significantly lower risk of macrovascular complications including cardiovascular disease and stroke [9], as well as atherosclerosis $[10,11]$. Long-term benefits of glycemic control have been demonstrated to extend to Type II diabetes mellitus. The UK Prospective Diabetes Study (UKPDS) that compared conventional dietary glucose control with intensive insulin therapy over 10 years initially reported decreased microvascular complications in the intensive therapy group [6]. In accordance with the DCCT-EDIC study, 10-year post-trial follow-up revealed lower incidence of macrovascular complications in the intensive therapy group compared to the conventional treatment group [12]. Overall, such findings indicate that previous episodes of poor glycemic control initiate the deleterious effects on the vasculature that persist despite intensive treatment and normalization of blood glucose. 


\section{In vivo evidence from animal models}

106 From observations arising from these clinical trials the term 'metabolic memory' was 107 introduced to describe the clinical features of diabetic complications that continue to develop 108 long after exposure to the hyperglycemic insult, despite improved glycemic control $[5,8,13]$. 109 Observations from pioneering studies in animal models also provide strong evidence of this 110 phenomenon. Early studies in dogs with alloxan-induced diabetes demonstrated persistent 111 retinopathy despite a period of 2.5 years of insulin therapy and glycemic control [14]. 112 Similarly sucrose-fed diabetic rats that received islet transplantation after 6 weeks of diabetes 113 exhibited reduced progression of retinopathy compared with islet transplantation following 11412 weeks of diabetes [15]. Another study in rats with streptozotocin-induced diabetes showed that retinal oxidative and nitrative stress resulted from periods of hyperglycemia, and that late reinstitution of strict glycemic control was insufficient to inhibit the progression of

117 diabetic retinopathy [16]. Initiation of good glycemic control after 2 months of hyperglycemic 118 conditions had only partial benefits with regard to oxidative and nitrative stress, whereas a 119 delay in this intervention for 6 months resulted in complete failure to reverse any 120 abnormalities [16]. These observations highlight difficulties in reversing hyperglycemia121 induced changes and the need for strict blood glucose control early in the progression of 122 diabetes. A study in mice cells revealed novel insights into the gene transcriptional changes 123 that underlie the phenomenon of metabolic memory with particular regard to inflammation. 124 Aortic endothelial cells from mice subjected to a hyperglycemic clamp for $3 \mathrm{~h}$ exhibited 125 increased expression of the pro-inflammatory $\mathrm{NF \kappa B}$ p65 subunit. This increase in gene 126 expression persisted for 6 days beyond normalization of blood glucose [17]. The p65-NFkB 127 transcription factor drives expression of several pro-inflammatory genes that contribute to 128 the development of diabetic vascular disease [18]. Furthermore p65 expression is increased 129 in diabetic ApoE-null mice aorta and appears to be a central mediator of inflammation in 130 diabetic complications [19].

\section{Cell culture experiments demonstrating persistence}

133 Transcriptional changes that govern the pathogenesis of diabetic complications in response to 134 the hyperglycemic insult have begun to be addressed in experiments performed in cell 135 culture. These studies have primarily focused on the vascular endothelium, a key site of 136 hypergylcemia-induced injury [20]. Persistent gene expression was addressed in an early 137 study of cultured primary human endothelial cells exposed to transient high glucose (HG) 
concentrations. Hyperglycemia induced expression of fibronectin and collagen IV that was maintained despite a return to normoglycemia [21]. Similarly, transient HG increased expression of p65 in primary endothelial cells. Consistent with observations in experimental animal models, the increase in transcription was maintained for up to 6 days following a return to physiological glycemic conditions [17]. Furthermore, expression of downstream p65-NFkB transactivation targets relevant to diabetic vascular injury, monocyte chemoattractant protein $1(M C P-1)$ and vascular cell adhesion molecule 1 (VCAM-1) were significantly increased and remained elevated upon return to normoglycemia [17]. The biochemical basis for the transcriptional response to HG has been delineated by key experiments that implicate the generation of reactive oxygen species (ROS) in the development and persistence of diabetic complications [22]. Increased expression of p65 and downstream transactivation targets was abolished by over-expression of either manganese superoxide dismutase (MnSOD) or uncoupling protein 1 (UCP-1) [17], both of which prevent hyperglycemia-induced superoxide accumulation [4,23]. Similarly the expression of protein markers of hyperglycemia such as protein kinase $C-\beta(\mathrm{PKC} \beta)$ and neutrophil cytosol factor 1 (p47phox) subunit of NADPH oxidase in endothelial and retinal cells was normalized by inhibition of ROS [24]. Thus the persistence of transcriptional changes following periods of hyperglycemia seems to be imparted by mitochondrial ROS overproduction. Linking these biochemical changes with nuclear mechanisms that govern transcriptional events is a key objective towards understanding glucose-induced transcriptional changes and metabolic memory. Several recent observations have sparked considerable interest in epigenetic mechanisms of gene regulation, particularly histone methylation, which could drive the sustained transcriptional changes observed under transient hyperglycemia.

\section{The role of chromatin modifications and epigenetic changes}

The term 'epigenetics' was traditionally employed to describe heritable changes in gene expression and cellular phenotype attributable to mechanisms other than alteration of the underlying nucleotide sequence of the DNA [25]. Recently this definition has evolved to include the study of transcriptional regulation with a particular focus on chromatin architecture and the ensuing/preceding interactions between non-genetic factors and chromatin. Chromatin is a dynamic complex of DNA, histone proteins, and numerous modifying complexes, that serves not only to efficiently package DNA, but also to provide a mechanism of transcriptional regulation [25]. Eukaryotic gene transcription is a precisely regulated and multifaceted process, and among other mechanisms is primarily controlled by 
172 the degree of chromatin accessibility. Altered states of transcription are initiated or 173 perpetuated by the dynamic structural adaptation of regions of chromatin to confer 174 transcriptionally permissive or repressive configurations [26]. This in turn regulates access 175 and subsequent enzymatic activity of the core transcriptional machinery and associated 176 factors to the underlying DNA sequence. Consequently, the structural state of chromatin 177 determines the transcriptional competency of a gene at a particular locus [27]. 178 Environmental factors are proposed to significantly influence the epigenetic signature, and 179 therefore the transcriptional competency of chromatin. Emerging evidence suggests a key 180 role for deregulated epigenetic transcriptional control in the pathogenesis of numerous 181 human diseases including metabolic and inflammatory disorders associated with diabetes. 182 The cellular transcriptional response to various environmental is exemplified by extensive 183 reports of epigenetic transcriptional changes in response to various stimuli and insults in cell 184 culture and experimental models. Thus exposure to environmental factors such as 185 hyperglycemia may lead to the establishment of altered transcriptional states of chromatin 186 that could potentially be retained for the lifetime of the organism. Furthermore there is 187 increasing evidence to indicate that in simple eukaryotes as well as animal models, epigenetic 188 transcriptional states can be meiotically inherited and trans-generationally persistent $[28,29]$.

\section{DNA methylation and diabetic complications}

191 A classical example of epigenetic transcriptional control is the covalent post-replicative 192 modification of DNA by the addition of methyl groups to cytosine residues primarily at CpG 193 dinucleotides within the genomic sequence, leading to transcriptional repression. Cytosine 194 methylation within DNA regulatory regions can inhibit transcription by physically precluding 195 the association of DNA and transcription factors [30] and influencing nucleosomal positioning

196 [31,32]. Methylated CpG residues are recognised and bound by several methyl-CpG binding 197 domain proteins which associate with various chromatin modifiers to establish 198 transcriptionally repressed chromatin [33-35]. Limited studies have focused on the role of 199 DNA methylation in the pathogenesis of diabetes, however altered methylation patterns have 200 been reported in patients with diabetes [36].

202 Regions of mammalian promoters enriched for CpG dinucleotides (CpG islands) are subject to 203 dynamic methyl modification during development [37]. Studies suggest that in utero 204 exposure to a poor nutritional environment might predispose the fetus to the development of 205 diabetes in adult life through aberrant DNA methylation in pancreatic islets of rats [38] and at 
the HNF4A gene locus of CD34+ stem cells from cord blood of neonates [39]. Importantly DNA methylation has been shown to play a role in regulation of insulin expression. Analysis of mouse embryonic stem cells revealed that DNA at the insulin (INS) promoter was methylated, and became demethylated as the cells differentiated into insulin expressing $\beta$-cells. Observation of this specific demethylation extended to the human insulin promoter of pancreatic $\beta$-cells [40].

Several studies have examined the potential role of DNA methylation in the development of diabetic complications, however the significance remains to be completely understood. One study demonstrated global DNA hypermethylation of peripheral blood leukocytes from patients with chronic kidney disease [41]. However analysis of kidney cells exposed to hyperglycemia and renal tissues from Type I diabetic rats showed no difference in DNA methylation of several candidate gene promoters [42]. Conversely numerous candidate genes linked to atherosclerosis were revealed to have altered DNA methylation patterns in endothelial cells [43]. With increased availability of high-throughput technologies for examining the DNA methylome, several observations relevant to diabetic complications have been highlighted by genome-wide studies. Genome-wide analysis of peripheral whole blood DNA methylation patterns from a cohort of 192 Type I diabetics recently identified 19 prospective $\mathrm{CpG}$ sites associated with the risk of diabetic nephropathy [44]. Global analysis of DNA methylation patterns in peripheral blood monocytes revealed significant changes in patients with increased risk for cardiovascular disease [45]. Furthermore, studies in smooth muscle cells of human atherosclerotic lesions and animal models including ApoE-null mice fed high-fat diets revealed associations between atherosclerosis and global DNA hypomethylation [46-48]. With regard to the development of Type I diabetes, recent genome-wide analysis of DNA methylation of monocytes from monozygotic twins discordant for Type I diabetes identified several diabetes-specific methylation variable positions. Further analysis revealed that the methylation patterns of some of these positions were altered in individuals prior to

\section{Chemical diversity of histone tail modifications}

237 Traditionally considered as primarily passive structural elements histone proteins are now 238 recognised as integral components of chromatin fundamental to the regulation of gene 239 expression [50,51]. Underlining their importance to nuclear regulation, histones are among 
240 the most highly conserved proteins through the eukaryotic domain [52]. Each histone

241 peptide possess an unstructured N-terminal tail composed of 15-44 amino acids that protrude 242 from the nucleosome to provide exposed surfaces for interaction with other proteins. The tail 243 domains harbor multiple lysine and arginine residues that are substrates for a variety of 244 covalent post-translational modifications including methylation, acetylation, phosphorylation 245 and ubiquitination [53]. Many of these modifications are associated with distinct transcriptional states and can generate synergistic or antagonistic effects in conjunction with other histone modifications to control transitions between transcriptionally active and inactive chromatin [27].

The wealth of post-translational modifications to the histone tail suggests a complicated and combinatory epigenetic language that co-ordinates structural and therefore transcriptional changes. Indeed several observations indicate that acetylation of particular histone tail residues can complement the effects of other histone modifications such as methylation on gene transcription [54]. Enzymatic transfer of an acetyl group to the $\varepsilon$-amino group of histone H3 and H4 lysine tails by histone acetyltransferases (HATs) has been correlated with transcriptional activation [55] most likely through alterations of electrostatic interactions [56]. By contrast, removal of the acetyl group by histone deacetylases (HDACs) is associated with transcriptional repression. Accordingly euchromatin is highly enriched for acetylated histones, whereas hypoacetylated histones are observed predominately at transcriptionally inactive heterochromatin $[57,58]$. Such broad observations have been proposed to reflect transcriptional competency rather than active transcription of such regions [59]. Nonetheless, hyperacetylation of certain histone lysine residues, particularly K9 and K14 of histone H3, has been thoroughly demonstrated as characteristic of promoter and enhancer regions of genes in active transcriptional states and several enzymes and complexes responsible for the addition and removal of the acetylation mark have been described [6063]. Recent studies have explored the relationship between histone hyperacetylation and gene expression under diabetic conditions. Cultured primary human endothelial cells exposed to hyperglycemic conditions displayed increased expression of p300 acetyltransferase and enrichment of this enzyme to the promoters of HG-responsive genes such as fibronectin [64]. Hyperacetylation of histone $\mathrm{H} 4$ at the promoter enhances expression of the INS gene [65]. Furthermore the $\beta$-cell specific transcription factor PDX1 was shown to interact with p300 to mediate proinsulin gene expression [66]. Thus histone acetylation is involved in both regulation of insulin secretion and damage associated with hyperglycemia. 
274 Therapeutic targeting of the enzymes that regulate these processes may therefore alleviate 275 some aspects of the disease. To this end the anti-inflammatory effects of HDAC inhibition

276 have recently been investigated. Genome-wide mRNA sequencing of human aortic endothelial cells revealed decreased expression of numerous inflammatory cytokines and chemokines such as $I L-6, I L-8, M C P-1$ and $M I F$ and cell adhesion molecule ICAM-1 upon treatment with the HDAC inhibitor Trichostatin A (Balcerczyk et al., manuscript in preparation). The aim of this review is not to provide an exhaustive description of each histone modification, which have recently been reviewed [67], but instead we focus on key relevant histone modifications in context to hyperglycemia, metabolic memory and its persistence. Perhaps the best characterized enzyme relevant to diabetic complications is the Set7 methyltransferase in primary culture experiments and small animal models of hyperglycemic memory.

\section{Histone methylation}

Histone methylation modifications catalysed by histone methyltransferases (HMTase) define and direct the formation of distinct chromatin regions [68,69]. Contrasting histone acetylation, methylation of lysine and arginine residues can be associated with both gene activation and repression, depending on the residue modified [69]. Most HMTase enzymes that specifically methylate lysine residues are classified as members of the SET family of proteins. Responsible for the methyltransferase activity is the presence of the evolutionarily conserved, 130-amino acid SET domain initially identified in three proteins shown to be required for maintenance of the expression of genes important to normal development in

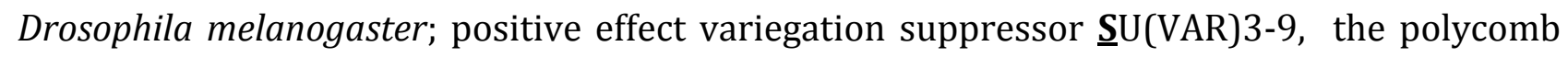
group protein Enhancer of zeste and the homeobox gene regulator Trithorax [70-73]. Identification of this common motif has rapidly increased the number of genetically and biochemically identified modifiers of histone proteins across several organisms. Multiple SET-domain HMTases have been characterized that methylate histone lysine residues in mammalian cells (Table 1). SET domain HMTases contribute to the regulation of transcriptional activity by methylating histone proteins within chromatin to maintain active and repressed transcriptional states through various nuclear processes. These modifications occur on several lysine residues within the N-terminal tail of histones H3 and H4, and the effect on transcription is dependent on both the specific lysine residue modified and the number of methyl groups covalently assigned. The enzymes responsible generally exhibit strong substrate and product specificity. Methylation of lysine residues 4, 36 and 79 of histone H3 are associated with active gene expression $[25,74,75]$, while methylated lysine 
residues 9 and 27 of histone $\mathrm{H} 3$ and lysine 20 of histone $\mathrm{H} 4$ are generally associated with transcriptionally silenced or inactive chromatin $[25,75,76]$. Recent genetic examination of genes encoding histone lysine methyltransferases in patients with Type 1 diabetes suggests 311 the importance of an inherited genetic component for the risk of diabetic complications. In 312 the well-characterised Finnish Diabetic Nephropathy Study (FinnDiane) cohort, a 313 polymorphism in the $S U V 39 H 2$ gene that encodes a H3K9-specific methyltransferase was 314 found to be associated with diabetic retinopathy [77]. Though this gene has not previously been linked to diabetic complications, it is a close homologue of SUV39H1 that has been 316 implicated in hyperglycemia-induced inflammation [17].

Table 1. Mammalian Histone Lysine Methyltransferase Enzymes

\begin{tabular}{|l|l|l|l|}
\hline \multirow{2}{*}{ Histone Target } & \multicolumn{3}{|c|}{ Lysine methyltransferase } \\
\cline { 2 - 4 } & Monomethylation & \multicolumn{1}{|c|}{ Dimethylation } & Trimethylation \\
\hline H3K4 & Set7 & $\begin{array}{l}\text { Smyd3, SETMAR, } \\
\text { NSD3 }\end{array}$ & $\begin{array}{l}\text { Smyd3, MLL, } \\
\text { SETD1A, SETD1B }\end{array}$ \\
\hline H3K9 & G9a, Eu-HMTase1 & G9a, Eu-HMTase1 & $\begin{array}{l}\text { SETDB1, SETDB2, } \\
\text { SUV39h1, } \\
\text { SUV39h2 }\end{array}$ \\
\hline H3K27 & EZH2, EZH1, G9a & $\begin{array}{l}\text { EZH2, EZH1, G9a, } \\
\text { NSD3 }\end{array}$ & EZH2, NSD3 \\
\hline H3K36 & NSD1, ASH1L & $\begin{array}{l}\text { NSD1, Smyd2, } \\
\text { SETMAR, ASH1L }\end{array}$ & $\begin{array}{l}\text { SETD2, ASH1L, } \\
\text { NSD2 }\end{array}$ \\
\hline H3K79 & DOT1L & DOT1L & DOT1L \\
\hline H4K20 & Pr-Set7(Set8), NSD2 & $\begin{array}{l}\text { Pr-Set7(Set8), } \\
\text { NSD1, SUV4-20 }\end{array}$ & NSD2, SUV4-20 \\
\hline
\end{tabular}


333 A SET-domain HMTase has recently received considerable attention for its ability to catalyse 334 the activating H3K4 monomethylation (H3K4me1) reaction. Sharing little sequence 335 homology with any of the six previously described yeast SET-domain containing proteins, the 336 enzyme was termed Set7 [78] (also described as Set9) [79]. Initial characterization demonstrated the ability of Set7 to stimulate GAL4-VP16-activated luciferase expression in cancer cell lines, implying a role in activator-induced transcription [79]. To this end, several functions attributable to Set7 activity have been observed in vitro which may be important to understanding the mechanism behind the transition between transcriptionally inactive and active chromatin. Set7-mediated H3K4 methylation inhibits Suv39h1-mediated H3K9 methylation and facilitates acetylation of both H3 and H4 by the acetyltransferase p300 [80]. Furthermore, association of the NuRD chromatin remodeling and deacetylase complex with the histone $\mathrm{H} 3$ tail is inhibited by H3K4 methylation, but not H3K9 methylation [79]. This antagonistic paradigm suggests that Set7-mediated H3K4me1 has the ability to maintain an active chromatin state by preventing both NuRD- and HP1-mediated (H3K9 methylationdependent) transcriptional silencing. Interestingly, methylation of H3K4 by Set7 did not have the same effect on H3K9 methylation by G9a [79]. This observation excludes Set7-mediated H3K4me1 as a global regulator of H3K9 methylation and by extension global transcriptional repression.

Set7 methyltransferase regulates metabolic memory and inflammatory diabetic complications

Several studies associate Set7 methyltransferase activity at H3K4 with gene activation under diabetic conditions in pancreatic cells [81-83], monocytes [84] and endothelial cells [17,85]. Recently HG concentrations were demonstrated to promote nuclear localization of Set7 in human endothelial cells [86]. Reduced H3K4me1 enrichment at discrete hyperglycemiaresponsive loci following Set7 depletion has been reported in models of hyperglycemic variability. Following transient exposure of endothelial cells to HG, persistent transcriptional activation of p65 (encoded by the RELA gene) and several inflammatory NFkB-dependent genes paralleling enrichment of Set7 and H3K4me1 at the RELA promoter was observed upon subsequent return to normoglycemia. This response was sustained for 6 days implying a memory of recent exposure to the metabolic insult. Set7 knockdown abrogated RELA promoter H3K4me1 enrichment and p65 mRNA and protein expression in response to 
transient and prior $\mathrm{HG}$ in both human and bovine endothelial cells. Accordingly the transcriptional persistence of p65 and its downstream transcriptional targets was attenuated $[17,85]$. Furthermore, sustained reduction of H3K9 methylation on the RELA promoter following transient and prior hyperglycemia was observed [85]. Methylation H3K9 and H3K4 have previously been demonstrated to inhibit each other [78] and the occlusion of H3K9 methylation by Set7-mediated methylation of H3K4 may represent another mechanism by which Set7 maintains an active chromatin state at the RELA promoter [85].

Experimental evidence suggests that Set7 may function as a co-activator of transcription through direct interaction with another transcriptional activator and specific co-recruitment to promoters. Monocyte transcription of a subset of TNF- $\alpha$-inducible NFKB-dependent inflammatory genes was compromised by depletion of endogenous Set7 through a different mechanism than previously reported for glucose-stimulated endothelial cells. Expression of TNF- $\alpha$-inducible p65-dependent MCP-1, IL-8 and TNF- $\alpha$ was shown to be dependent on Set7 expression [84]. TNF- $\alpha$-stimulated cells displayed increased MCP-1 expression concomitant with enrichment of Set7, p65 and H3K4 methylation at the MCP-1 and TNF- $\alpha$ promoters. Targeted Set7 disruption using shRNA impaired this response, namely the promoter-specific recruitment of p65. Microarray analysis of TNF- $\alpha$-stimulated Set7 knockdown monocytes revealed altered transcriptional induction of over $25 \%$ of TNF- $\alpha$-regulated genes. Interestingly, the level of p65 expression detected by western blot was similar across control and Set7 deficient cells [84]. This observation indicates that the effects on downstream transcription targets were not a result of attenuated RELA transcriptional activation, as was the case in the response of endothelial cells to glucose stimulation. Rather Set7 appears to act as a co-activator of a subset of p65-mediated transcription of TNF- $\alpha$-sensitive genes. While these seemingly discordant findings may reflect differences across cell types, it is likely that

Intriguingly the p65 subunit was recently identified as a novel substrate for Set7-mediated methylation. Studies have demonstrated this modification to have implications for p65 transactivity and downstream transcription of $\mathrm{NF}_{\kappa} \mathrm{B}$-dependent genes. This is a significant finding given the enzymes's reported role in persistent transcriptional activation of the RELA promoter in endothelial cells, and further implicates Set7 in regulation of NFkB-dependent methylation by a single point mutation at K37, indicating this residue to be the lysine targeted 
400 for modification [87]. Methylation dependent regulation of p65 may represent an integral control point with regard to the expression of a variety of genes.

While several known examples of posttranslational p65 modification exist, the biological response to Set7-mediated methylation of p65 remains to be fully established. Relative to the unmodified protein, p65 methylated at K37 is restricted to the nucleus and enriched at a subset of NFkB-dependent promoters in response to TNF-a stimulation [87]. Conversely, examples of this modification have also linked Set7 to transcriptional repression. Two alternative sites at K315 and K316 that are subject to Set7-dependent methylation have been reported, modification of which can result in negative regulation of p65 transactivity in vivo [88]. Subsequent kinetic analysis reveals that the K37 methylation event occurs prior to K315 411 and K316 methylation, and may be the preferential methylation site [87]. Nevertheless p65 appears to contain Set7-dependent regulatory sites for both transcriptional activation and repression of downstream gene targets. Overall Set7 appears to be a key mediator of the proinflammatory response to hyperglycemia through both increased p65 expression via promoter H3K4 methylation enrichment [17] and post-translational methylation of distinct lysine residues within the p65 subunit of $\mathrm{NF}_{\kappa} B[87,88]$. In support of Set7-dependent regulation of p65 transactivity, recent massive parallel sequencing analysis of a model of Set7 depletion in human endothelial cells implicates deregulated p65 transactivity in the differential expression of numerous genes, some of which have associations with

A similar co-activational role has been described in pancreatic $\beta$-cells with regard to Set7 recruitment and enrichment of $\mathrm{H} 3 \mathrm{~K} 4$ methylation at the INS promoter. Coimmunoprecipitation and GST pull-down assays in conjunction with ChIP assays demonstrate the recruitment of Set7 and subsequent methyltransferase activity to be mediated through direct interaction with transcriptional activator; pancreatic duodenal homeobox-1 (PDX-1). Depletion of either Set7 or PDX-1 expression significantly decreased transcription of a reporter construct driven by an intact INS promoter [83]. Significant reduction in transcription of a subset of glucose responsive genes was observed in mouse $\beta$-cells and primary islets in vitro, following siRNA-mediated Set7 depletion [81]. Unexpectedly, this transcriptional diminution correlated with reduced H3K4me2 enrichment at the proximal promoter regions of Ins1, Ins2 and Slc2a2, while no change to the H3K4me1 status was reported. The authors noted that these results do not necessarily rule out Set7's function as a 
monomethylase and raise the possibility of another closely linked HMTase capable of completing the di-methylation reaction on the enzymatic product of Set7 [81]. Importantly the overall experimental observations highlight the necessity of Set7 function in $\beta$-cells for proper insulin production and secretion.

A recent study implicates Set7 as a co-activator of TGF- $\beta$-induced expression of extracellular matrix genes relevant to the development of diabetic nephropathy [89]. Under hyperglycemic conditions, increased expression of fibrotic genes Col1a1, CTGF and PAI-1 in rat mesangial cells was demonstrated to correlate with enrichment of promoter H3K4me1 and Set7 recruitment. Increased gene expression and Set7 recruitment were attenuated by both pretreatment with a TGF- $\beta$-specific antibody and targeted Set7 disruption by siRNA transfection [89]. Col1a1 [90], CTGF [91] and PAI-1 [92] are bona fide transactivation targets of SMAD3, a key mediator of renal fibrosis and inflammation [91,93,94]. Indeed deletion of SMAD3 conferred protection against fibrogenesis in a rodent model of diabetic nephropathy [95]. Under diabetic conditions, critical mediators of diabetic complications AGEs [91,96] and Angiotensin II $[93,97]$ can also activate SMAD signaling pathways to stimulate extracellular matrix production via TGF- $\beta$-dependent and independent mechanisms. Thus it is tempting to speculate a mediatory role for SMAD3 in the recruitment and specific enrichment of Set7 to activating genes targets in response to TGF- $\beta$ stimulation to potentiate the H3K4me1 reaction. Interaction between Set7 and SMAD3 has not been demonstrated experimentally, but may provide another example of transcription factor-Set7 co-activation possibly via a methylationdependent mechanism. Disruption of a potential Set7/SMAD3 interaction may provide a novel therapeutic target to alleviate renal complications associated with the diabetic milieu. Taken together, these findings provide evidence of co-recruitment of Set7 to specific genes and suggest that Set7 may function in complex with transcriptional co-activators. For instance ordered recruitment of Set7 and other transcriptional regulatory proteins indicate that Set7 co-assembles as a component of a pre-initiation complex required for transcriptional activation of collagenase I [98].

\section{Set7 methylates non-histone substrates}

As highlighted by reports of Set7-dependent post-translational methylation of p65 lysine residues, the molecular events mediated by Set7 in response to hyperglycemia might not be restricted to histone methylation. Analysis of Set7 methyltransferase activity toward lysine residues of non-histone proteins has revealed previously unknown mechanisms by which 
Set7 may contribute to transcriptional regulation and other cellular processes [99].

469 Numerous additional substrates have recently been described sparking considerable interest 470 in the characterization of these methylation events and the identification of novel lysine 471 targets [100]. Adding to the complexity of the role of Set7 in transcriptional regulation, 472 transcription factors predominate the list of confirmed Set7 substrates. The in vivo 473 consequences of post-translational methylation on most of these targets remain to be fully understood. However numerous observations of Set7 methyltransferase activity-dependent transcription factor stability and activity have been reported, and several examples are described in Figure 2.

Recent studies suggest novel mechanisms of Set7 recruitment to individual promoters by Set7-transcription factor binding and modification, providing further evidence of a coactivational role. Several recently identified Set7 substrates display modified biological activity following Set7-mediated methylation including p53 [101], DNMT1 [102] and ER- $\alpha$ [103]. This post-translational methylation can result in altered expression of downstream transcriptional targets. For instance the TFIID transcription factor complex component TAF10 is methylated at K189 by Set7 [104]. While this modification does not affect the incorporation of TAF10 into TFIID, methylated TAF10 displays increased affinity for RNAPII suggesting a role for this modification in regulating preinitiation complex formation. To this end, transcription of several known TAF10-dependent genes was enhanced by methylation of TAF10 [104]. While this report highlights an example mechanism whereby Set7 is brought within close proximity of an activating promoter, subsequent effects on H3K4 methylation remain unresolved.

Sequential Set7 recruitment to histone H3 following TAF10 promoter binding remains to be demonstrated. Recent reports suggest this indeed to be the case with an interaction between Set7 and another recently characterized substrate. STAT3 is methylated at K140 by Set7, and de-methylated by LSD1 [105]. This modification both positively and negatively regulates the expression of a multitude of STAT3-dependent genes in response to IL-6 stimulation, and can be disrupted by Set7 depletion or cells expressing STAT3 R140 mutant proteins. Furthermore, STAT3 promoter occupancy of a subset of these genes was shown to be dependent on Set7 methyltransferase activity. This regulation is promoter specific as not all STAT3-dependent IL-6 responsive promoters exhibited methylated STAT3 enrichment [105]. IL-6 is considered to play a central role in the development of diabetic complications 
502 including diabetic nephropthy [106,107]. Circulating IL-6 levels are reportedly elevated in 503 patients with type 2 diabetes [108], are associated with poor glycemic control [109]. Recent 504 microarray analysis of diabetic kidneys revealed significant up-regulation of genes in the 505 JAK/STAT pathway within glomeruli and tubules [110]. Furthermore components of the 506 diabetic milieu including ROS [111], angiotensin II signaling [105] and AGEs [112] have been 507 experimentally demonstrated to activate this pathway in kidney cells. In a study of diabetic 508 mice with compromised STAT3 activity, extracellular matrix-associated TGF- $\beta$ and type IV 509 collagen expression as well as mesangial cell proliferation were inhibited. Attenuation of the 510 diseased phenotype extended to a reduction in the expression of pro-inflammatory factors IL511 6, ICAM-1, MCP-1 and nuclear translocation of $\mathrm{NF}_{\kappa} B$ [113]. Given the responsivity of Set7 to 512 hyperglycemia and ROS and its transactivity-modifying capacity of STAT3, it is reasonable to 513 speculate a role for the methyltransferase in the development and progression of diabetic 514 nephropathy through this pathway.

516 The SOCS3 gene was strongly up-regulated following Set7 knockdown, and displays increased 517 STAT3 enrichment at the promoter [105]. Furthermore it was demonstrated that Set7 is 518 recruited to the SOCS3 promoter after STAT3 recruitment. This sequence of events may 519 function to clear STAT3 from the activating promoter and simultaneously potentiate 520 methylation of H3K4 [105]. Set7/STAT3-regulated expression of SOCS3 may hold therapeutic 521 relevance for diabetic nephropathy as SOCS3 is a negative-feedback regulator of the 522 JAK/STAT pathway [114], specifically STAT3 activation [115]. To this end, over-expression of 523 SOCS3 inhibited the hyperglycemia-induced activation of STAT-responsive inflammatory 524 genes $M C P-1, I C A M-1$ and $I L-6$ [116].

526 A similar interaction between Set7 and the androgen receptor (AR) has been described [117] 527 as a methylation event important for AR activation and recruitment to androgen-regulated 528 promoters. This interaction may facilitate H3K4me1, as depletion of endogenous Set7 in 529 cancer cells reduced H3K4me1 enrichment at proximal and enhancer regions of the 530 androgen-dependent PSA promoter [117]. This observation holds relevance for diabetic 531 complications, as hyperandrogenemia is associated with $\beta$-cell dysfunction $[118,119]$. In 532 support, a recent study in a mouse model reported increased systemic oxidative stress 533 stemming from testosterone treatment was AR-dependent. Furthermore female mouse 534 pancreatic islets treated with streptozotocin displayed increased $\beta$-cell destruction [120]. 
535 Further investigation may reveal Set7 as a potential therapeutic target in the treatment of 536 hyperandrogenemia and the accompanying insulin resistance.

\section{Conclusion}

539 Estimates indicate that the incidence of diabetes mellitus and associated complications is 540 rapidly increasing. It is therefore imperative that strategies to combat progression of 541 detrimental complications are established. Knowledge of the molecular etiology of diabetes mellitus is paramount to understanding the link between environmental factors such as nutrition and altered/persistent gene expression that drive the disease phenotype. Clinical and experimental studies have revealed the deleterious consequences of exposure to hyperglycemia, and clearly highlight the importance of strict glycemic control. The concept that a cell retains a metabolic memory of prior exposure to hyperglycemic events was a seminal hypothesis that arose from epidemiological studies of diabetic patients. This concept is further exemplified by experimental studies that demonstrate hyperglycemia-induced changes in gene expression that persist despite a return to physiological glucose concentrations.

The dynamic processes that govern epigenetic regulation of chromatin confer an additional layer of transcriptional control that links gene expression with environmental signalling. Experimental evidence demonstrates that hyperglycemia can induce epigenetic modifications to chromatin structure via various nuclear processes. Specific histone-modifying enzymes have been implicated, and have been shown to regulate the expression of inflammatory genes in cells of vascular origin. In these models of glycemic memory, the Set7 methyltransferase was shown to engage the histone $\mathrm{H} 3$ tail to establish H3K4me1 at the pro-inflammatory RELA promoter, a modification that persists despite normalisation of glucose conditions. Certainly other histone modifications and the responsible enzymes are expected to be involved in transcriptional changes induced by elevated glucose concentrations, and are likely to be explored in models of glycemic memory. Characterisation of these events will be greatly assisted by recent advances in powerful genome-wide technologies such as massive parallel sequencing and bioinformatics tools. For instance genome-wide analysis of primary vascular cells exposed to HG concentrations has revealed relationships between acetylated histone tails, DNA methylation and expression of genes associated with endothelial dysfunction [121]. 
568 The role of Set7 in gene activational events under diabetic conditions extends to pancreatic 569 and monocytic transcriptional regulation that also hold relevance for diabetic complications. 570 Additionally, recent and rapid expansion of substrates alternative to the histone H3 tail may 571 provide further evidence of the enzyme's involvement in the pathogenesis of diabetic 572 complications. Defining non-histone Set7 substrates is critical to understanding the role of Set7 in 573 transcriptional regulation in health and disease. This avenue of investigation may uncover novel 574 mechanisms by which Set7 is recruited to activating promoters at specific loci to execute the H3K4 methylation reaction. By the same token, Set7-regulated protein activity and stability, and consequential effects on transactivation potential is another mechanism of transcriptional control that may be applicable to diabetic complications. To attenuate the burden of diabetic complications it may soon be possible to interrupt the epigenetic pathways that promote diabetic complications such as vascular injury. Specific targeting of the enzymes implicated in chromatin structure and gene function such as Set7 provides potential for future epigenetic therapy for diabetic complications. However several challenges to this approach are apparent in the multiplicity of modifications that govern gene expression. The combinatorial nature of histone modification patterns at individual loci and genome-wide will be rapidly unveiled with advances in sequencing technology. It is anticipated that this will strongly build upon key findings from in vitro studies of glucose- and ROS-induced vascular injury and potentially lead to therapeutic inhibition of methyl-writing and methyl-erasing enzymes to attenuate complications of diabetes.

Another strategy to abrogate the effects of hyperglycemic exposure is inhibition of ROS overproduction. Oxidative stress plays a pivotal role in the development of complications of diabetes by activating numerous pathogenic pathways [122]. Furthermore increased ROS production inactivates 2 athero-protective enzymes eNOS [123,124] and prostacyclin synthase [125]. The significant reduction of eNOS and prostacyclin synthase activity attributable to hyperglycemia-induced ROS overproduction was attenuated by treatment with a superoxide dismutase/catalase mimetic [23]. Similar strategies of ROS inhibition have been demonstrated to prevent the development of complications including cardiomyopathy $[126,127]$, nephropathy [128,129], retinopathy [130] and neuropathy [131] in diabetic mice. 
602

603

604

605

606

607 
Figure 1 Massive parallel sequencing analysis of a model of Set7 depletion in human numerous genes, some of which have associations with inflammation and diabetic complications. Vertical lines categorise the cellular location of protein products of the deregulated genes in the network: extracellular, membrane, cytoplasm, nucleus and unspecified. The single arrow going toward p65 marks the interaction with the Set7 methyltransferase. Red circles in the right corner of a gene indicate increased expression and blue circles indicate decreased expression. This transcription factor centric network was generated using the commercial software MetaCore $^{\mathrm{TM}}$ version 6.8 build 30387 (GeneGo, Inc) which draws from the manually curated MetaCore database. 
Figure 2 Examples of transcription-associated protein substrates for the methyltransferase reaction catalysed by Set7. Several of these modifications may contribute to a positive transcriptional outcome. Set7-mediated methylation of K142 destabilizes DNMT1 protein by facilitating polyubiquitination and subsequent proteasomemediated degradation [102]. By contrast methylation of p53 at K372 and subsequent acetylation by Tip60 acetyltransferase results in increased stability of the p53 protein, thereby influencing p53-mediated gene expression [132]. Methylation of p65 at K37 methylated p65 is restricted to the nucleus and is enriched at a subset of $\mathrm{NF \kappa B}$-dependent 


\section{Sources of Funding}

698 The authors acknowledge grant and fellowship support from the Juvenile Diabetes Research 699 Foundation International (JDRF), the Diabetes Australia Research Trust (DART), the National 700 Health and Medical Research Council (NHMRC) and the National Heart Foundation of 701 Australia (NHF). STK is supported by an Australian Postgraduate Award (APA). AE-O is a 702 Senior Research Fellow supported by the NHMRC. Supported in part by the Victorian Government's Operational Infrastructure Support Program.

Declarations

707

None 


\section{References}

1. Federation ID. IDF Diabetes Atlas. 4 ed. Brussles, Belgium: International Diabetes Federation; 2009.

2. Shaw JE, Chisholm DJ. 1: Epidemiology and prevention of type 2 diabetes and the metabolic syndrome. Med J Aust. 2003 Oct 6;179(7):379-83.

3. El-Osta A. Glycemic memory. Curr Opin Lipidol. 2012 Feb;23(1):24-9.

4. Brownlee M. Biochemistry and molecular cell biology of diabetic complications. Nature. 2001 Dec 13;414(6865):813-20.

5. The effect of intensive treatment of diabetes on the development and progression of long-term complications in insulin-dependent diabetes mellitus. The Diabetes Control and Complications Trial Research Group. N Engl J Med. 1993 Sep 30;329(14):977-86.

6. UK Prospective Diabetes Study (UKPDS). VIII. Study design, progress and performance. Diabetologia. 1991 Dec;34(12):877-90.

7. The Diabetes Control and Complications Trial (DCCT). Design and methodologic considerations for the feasibility phase. The DCCT Research Group. Diabetes. 1986 May;35(5):530-45.

8. Sustained effect of intensive treatment of type 1 diabetes mellitus on development and progression of diabetic nephropathy: the Epidemiology of Diabetes Interventions and Complications (EDIC) study. JAMA. 2003 Oct 22;290(16):2159-67.

9. Nathan DM, Cleary PA, Backlund JY, et al. Intensive diabetes treatment and cardiovascular disease in patients with type 1 diabetes. N Engl J Med. 2005 Dec 22;353(25):2643-53.

10. Cleary PA, Orchard TJ, Genuth S, et al. The effect of intensive glycemic treatment on coronary artery calcification in type 1 diabetic participants of the Diabetes Control and Complications Trial/Epidemiology of Diabetes Interventions and Complications (DCCT/EDIC) Study. Diabetes. 2006 Dec;55(12):3556-65.

11. Nathan DM, Lachin J, Cleary $\mathbf{P}$, et al. Intensive diabetes therapy and carotid intimamedia thickness in type 1 diabetes mellitus. N Engl J Med. 2003 Jun 5;348(23):2294303.

12. Holman RR, Paul SK, Bethel MA, Matthews DR, Neil HA. 10-year follow-up of intensive glucose control in type 2 diabetes. N Engl J Med. 2008 Oct 9;359(15):157789.

13. Effect of intensive therapy on the microvascular complications of type 1 diabetes mellitus. JAMA. 2002 May 15;287(19):2563-9.

14. Engerman RL, Kern TS. Progression of incipient diabetic retinopathy during good glycemic control. Diabetes. 1987 Jul;36(7):808-12.

15. Hammes HP, Klinzing I, Wiegand S, Bretzel RG, Cohen AM, Federlin K. Islet transplantation inhibits diabetic retinopathy in the sucrose-fed diabetic Cohen rat. Invest Ophthalmol Vis Sci. 1993 May;34(6):2092-6.

16. Kowluru RA. Effect of reinstitution of good glycemic control on retinal oxidative stress and nitrative stress in diabetic rats. Diabetes. 2003 Mar;52(3):818-23.

17. El-Osta A, Brasacchio D, Yao D, et al. Transient high glucose causes persistent epigenetic changes and altered gene expression during subsequent normoglycemia. J Exp Med. 2008 Sep 29;205(10):2409-17.

18. Thurberg BL, Collins T. The nuclear factor-kappa B/inhibitor of kappa B autoregulatory system and atherosclerosis. Curr Opin Lipidol. 1998 Oct;9(5):387-96.

19. Lewis P, Stefanovic N, Pete J, et al. Lack of the antioxidant enzyme glutathione peroxidase- 1 accelerates atherosclerosis in diabetic apolipoprotein E-deficient mice. Circulation. 2007 Apr 24;115(16):2178-87. 
20. Bakker W, Eringa EC, Sipkema P, van Hinsbergh VW. Endothelial dysfunction and diabetes: roles of hyperglycemia, impaired insulin signaling and obesity. Cell Tissue Res. 2009 Jan;335(1):165-89.

21. Roy S, Sala R, Cagliero E, Lorenzi M. Overexpression of fibronectin induced by diabetes or high glucose: phenomenon with a memory. Proc Natl Acad Sci U S A. 1990 Jan;87(1):404-8.

22. Nishikawa T, Edelstein D, Du XL, et al. Normalizing mitochondrial superoxide production blocks three pathways of hyperglycaemic damage. Nature. $2000 \mathrm{Apr}$ 13;404(6779):787-90.

23. Brownlee M. The pathobiology of diabetic complications: a unifying mechanism. Diabetes. 2005 Jun;54(6):1615-25.

24. Ihnat MA, Thorpe JE, Kamat CD, et al. Reactive oxygen species mediate a cellular 'memory' of high glucose stress signalling. Diabetologia. 2007 Jul;50(7):1523-31.

25. Turner BM. Cellular memory and the histone code. Cell. 2002 Nov 1;111(3):285-91.

26. Bird A. Perceptions of epigenetics. Nature. 2007 May 24;447(7143):396-8.

27. Jenuwein T, Allis CD. Translating the histone code. Science. 2001 Aug 10;293(5532):1074-80.

28. Greer EL, Maures TJ, Ucar D, et al. Transgenerational epigenetic inheritance of longevity in Caenorhabditis elegans. Nature. 2011 Nov 17;479(7373):365-71.

29. Braunschweig M, Jagannathan V, Gutzwiller A, Bee G. Investigations on transgenerational epigenetic response down the male line in $\mathrm{f} 2$ pigs. PLoS One. 2012;7(2):e30583.

30. Iguchi-Ariga SM, Schaffner W. CpG methylation of the cAMP-responsive enhancer/promoter sequence TGACGTCA abolishes specific factor binding as well as transcriptional activation. Genes Dev. 1989 May;3(5):612-9.

31. Pennings S, Allan J, Davey CS. DNA methylation, nucleosome formation and positioning. Brief Funct Genomic Proteomic. 2005 Feb;3(4):351-61.

32. Chodavarapu RK, Feng S, Bernatavichute YV, et al. Relationship between nucleosome positioning and DNA methylation. Nature. 2010 Jul 15;466(7304):388-92.

33. Fuks F, Hurd PJ, Wolf D, Nan X, Bird AP, Kouzarides T. The methyl-CpG-binding protein MeCP2 links DNA methylation to histone methylation. J Biol Chem. 2003 Feb 7;278(6):4035-40.

34. Meehan RR, Lewis JD, Bird AP. Characterization of MeCP2, a vertebrate DNA binding protein with affinity for methylated DNA. Nucleic Acids Res. 1992 Oct 11;20(19):508592.

35. Zhang Y, Ng HH, Erdjument-Bromage H, Tempst P, Bird A, Reinberg D. Analysis of the NuRD subunits reveals a histone deacetylase core complex and a connection with DNA methylation. Genes Dev. 1999 Aug 1;13(15):1924-35.

36. Maier S, Olek A. Diabetes: a candidate disease for efficient DNA methylation profiling. J Nutr. 2002 Aug;132(8 Suppl):2440S-3S.

37. Reik W, Dean W, Walter J. Epigenetic reprogramming in mammalian development. Science. 2001 Aug 10;293(5532):1089-93.

38. Thompson RF, Fazzari MJ, Niu H, Barzilai N, Simmons RA, Greally JM. Experimental intrauterine growth restriction induces alterations in DNA methylation and gene expression in pancreatic islets of rats. J Biol Chem. 2010 May 14;285(20):15111-8.

39. Einstein F, Thompson RF, Bhagat TD, et al. Cytosine methylation dysregulation in neonates following intrauterine growth restriction. PLoS One. 2010;5(1):e8887.

40. Kuroda A, Rauch TA, Todorov I, et al. Insulin gene expression is regulated by DNA methylation. PLoS One. 2009;4(9):e6953. 
41. Stenvinkel P, Karimi M, Johansson S, et al. Impact of inflammation on epigenetic DNA methylation - a novel risk factor for cardiovascular disease? J Intern Med. 2007 May;261(5):488-99.

42. Brennan EP, Ehrich M, O'Donovan H, et al. DNA methylation profiling in cell models of diabetic nephropathy. Epigenetics. 2010 Jul 1;5(5):396-401.

43. Chan Y, Fish JE, D'Abreo C, et al. The cell-specific expression of endothelial nitricoxide synthase: a role for DNA methylation. J Biol Chem. 2004 Aug 13;279(33):35087100.

44. Bell CG, Teschendorff AE, Rakyan VK, Maxwell AP, Beck S, Savage DA. Genomewide DNA methylation analysis for diabetic nephropathy in type 1 diabetes mellitus. BMC Med Genomics. 2010;3:33.

45. Kim M, Long TI, Arakawa K, Wang R, Yu MC, Laird PW. DNA methylation as a biomarker for cardiovascular disease risk. PLoS One. 2010;5(3):e9692.

46. Hiltunen MO, Turunen MP, Hakkinen TP, et al. DNA hypomethylation and methyltransferase expression in atherosclerotic lesions. Vasc Med. 2002 Feb;7(1):5-11.

47. Hiltunen MO, Yla-Herttuala S. DNA methylation, smooth muscle cells, and atherogenesis. Arterioscler Thromb Vasc Biol. 2003 Oct 1;23(10):1750-3.

48. Laukkanen MO, Mannermaa S, Hiltunen MO, et al. Local hypomethylation in atherosclerosis found in rabbit ec-sod gene. Arterioscler Thromb Vasc Biol. 1999 Sep;19(9):2171-8.

49. Rakyan VK, Beyan H, Down TA, et al. Identification of type 1 diabetes-associated DNA methylation variable positions that precede disease diagnosis. PLoS Genet. 2011 Sep;7(9):e1002300.

50. Luger K. Dynamic nucleosomes. Chromosome Res. 2006;14(1):5-16.

51. Strahl BD, Allis CD. The language of covalent histone modifications. Nature. 2000 Jan 6;403(6765):41-5.

52. Malik HS, Henikoff S. Phylogenomics of the nucleosome. Nat Struct Biol. 2003 Nov;10(11):882-91.

53. Kouzarides T. Chromatin modifications and their function. Cell. $2007 \mathrm{Feb}$ 23;128(4):693-705.

54. An W. Histone acetylation and methylation: combinatorial players for transcriptional regulation. Subcell Biochem. 2007;41:351-69.

55. Roth SY, Denu JM, Allis CD. Histone acetyltransferases. Annu Rev Biochem. 2001;70:81-120.

56. Shogren-Knaak M, Ishii H, Sun JM, Pazin MJ, Davie JR, Peterson CL. Histone H4-K16 acetylation controls chromatin structure and protein interactions. Science. $2006 \mathrm{Feb}$ 10;311(5762):844-7.

57. Braunstein M, Rose AB, Holmes SG, Allis CD, Broach JR. Transcriptional silencing in yeast is associated with reduced nucleosome acetylation. Genes Dev. 1993 Apr;7(4):592-604.

58. Grunstein M. Histone acetylation in chromatin structure and transcription. Nature. 1997 Sep 25;389(6649):349-52.

59. Eberharter A, Becker PB. Histone acetylation: a switch between repressive and permissive chromatin. Second in review series on chromatin dynamics. EMBO Rep. 2002 Mar;3(3):224-9.

60. Roh TY, Cuddapah S, Zhao K. Active chromatin domains are defined by acetylation islands revealed by genome-wide mapping. Genes Dev. 2005 Mar 1;19(5):542-52.

61. Roh TY, Wei G, Farrell CM, Zhao K. Genome-wide prediction of conserved and nonconserved enhancers by histone acetylation patterns. Genome Res. 2007 Jan;17(1):74-81. 
62. de Ruijter AJ, van Gennip AH, Caron HN, Kemp S, van Kuilenburg AB. Histone deacetylases (HDACs): characterization of the classical HDAC family. Biochem J. 2003 Mar 15;370(Pt 3):737-49.

63. Wade PA, Wolffe AP. Histone acetyltransferases in control. Curr Biol. $1997 \mathrm{Feb}$ 1;7(2):R82-4.

64. Chen S, Feng B, George B, Chakrabarti R, Chen M, Chakrabarti S. Transcriptional coactivator p300 regulates glucose-induced gene expression in endothelial cells. Am J Physiol Endocrinol Metab. 2010 Jan;298(1):E127-37.

65. Mosley AL, Ozcan S. Glucose regulates insulin gene transcription by hyperacetylation of histone h4. J Biol Chem. 2003 May 30;278(22):19660-6.

66. Mosley AL, Corbett JA, Ozcan S. Glucose regulation of insulin gene expression requires the recruitment of $\mathrm{p} 300$ by the beta-cell-specific transcription factor Pdx-1. Mol Endocrinol. 2004 Sep;18(9):2279-90.

67. Suganuma T, Workman JL. Signals and combinatorial functions of histone modifications. Annu Rev Biochem. 2011 Jun 7;80:473-99.

68. Cheung P, Lau P. Epigenetic regulation by histone methylation and histone variants. Mol Endocrinol. 2005 Mar;19(3):563-73.

69. Zhang Y, Reinberg D. Transcription regulation by histone methylation: interplay between different covalent modifications of the core histone tails. Genes Dev. 2001 Sep 15;15(18):2343-60.

70. Qian C, Zhou MM. SET domain protein lysine methyltransferases: Structure, specificity and catalysis. Cell Mol Life Sci. 2006 Dec;63(23):2755-63.

71. Tschiersch B, Hofmann A, Krauss V, Dorn R, Korge G, Reuter G. The protein encoded by the Drosophila position-effect variegation suppressor gene Su(var)3-9 combines domains of antagonistic regulators of homeotic gene complexes. EMBO J. 1994 Aug 15;13(16):3822-31.

72. Stassen MJ, Bailey D, Nelson S, Chinwalla V, Harte PJ. The Drosophila trithorax proteins contain a novel variant of the nuclear receptor type DNA binding domain and an ancient conserved motif found in other chromosomal proteins. Mech Dev. 1995 Aug;52(2-3):209-23.

73. Jenuwein T, Laible G, Dorn R, Reuter G. SET domain proteins modulate chromatin domains in eu- and heterochromatin. Cell Mol Life Sci. 1998 Jan;54(1):80-93.

74. Sims RJ, 3rd, Nishioka K, Reinberg D. Histone lysine methylation: a signature for chromatin function. Trends Genet. 2003 Nov;19(11):629-39.

75. Boggs BA, Cheung P, Heard E, Spector DL, Chinault AC, Allis CD. Differentially methylated forms of histone $\mathrm{H} 3$ show unique association patterns with inactive human X chromosomes. Nat Genet. 2002 Jan;30(1):73-6.

76. Noma K, Allis CD, Grewal SI. Transitions in distinct histone H3 methylation patterns at the heterochromatin domain boundaries. Science. 2001 Aug 10;293(5532):1150-5.

77. Syreeni A, El-Osta A, Forsblom C, et al. Genetic examination of SETD7 and SUV39H1/H2 methyltransferases and the risk of diabetes complications in patients with type 1 diabetes. Diabetes. 2011 Nov;60(11):3073-80.

78. Wang H, Cao R, Xia L, et al. Purification and functional characterization of a histone H3-lysine 4-specific methyltransferase. Mol Cell. 2001 Dec;8(6):1207-17.

79. Nishioka K, Chuikov S, Sarma K, et al. Set9, a novel histone H3 methyltransferase that facilitates transcription by precluding histone tail modifications required for heterochromatin formation. Genes Dev. 2002 Feb 15;16(4):479-89.

80. Zegerman P, Canas B, Pappin D, Kouzarides T. Histone H3 lysine 4 methylation disrupts binding of nucleosome remodeling and deacetylase (NuRD) repressor complex. J Biol Chem. 2002 Apr 5;277(14):11621-4. 
81. Deering TG, Ogihara T, Trace AP, Maier B, Mirmira RG. Methyltransferase Set7/9 maintains transcription and euchromatin structure at islet-enriched genes. Diabetes. 2009 Jan;58(1):185-93.

82. Ogihara T, Vanderford NL, Maier B, Stein RW, Mirmira RG. Expression and function of Set7/9 in pancreatic islets. Islets. 2009 Nov-Dec;1(3):269-72.

83. Francis J, Chakrabarti SK, Garmey JC, Mirmira RG. Pdx-1 links histone H3-Lys-4 methylation to RNA polymerase II elongation during activation of insulin transcription. J Biol Chem. 2005 Oct 28;280(43):36244-53.

84. Li Y, Reddy MA, Miao F, et al. Role of the histone H3 lysine 4 methyltransferase, SET7/9, in the regulation of NF-kappaB-dependent inflammatory genes. Relevance to diabetes and inflammation. J Biol Chem. 2008 Sep 26;283(39):26771-81.

85. Brasacchio D, Okabe J, Tikellis C, et al. Hyperglycemia induces a dynamic cooperativity of histone methylase and demethylase enzymes associated with geneactivating epigenetic marks that coexist on the lysine tail. Diabetes. 2009 May;58(5):1229-36.

86. Okabe J, Orlowski C, Balcerczyk A, et al. Distinguishing Hyperglycemic Changes by Set7 in Vascular Endothelial Cells. Circ Res. 2012 Mar 8.

87. Ea CK, Baltimore D. Regulation of NF-kappaB activity through lysine monomethylation of p65. Proc Natl Acad Sci U S A. 2009 Nov 10;106(45):18972-7.

88. Yang XD, Huang B, Li M, Lamb A, Kelleher NL, Chen LF. Negative regulation of NFkappaB action by Set9-mediated lysine methylation of the RelA subunit. EMBO J. 2009 Apr 22;28(8):1055-66.

89. Sun G, Reddy MA, Yuan H, Lanting L, Kato M, Natarajan R. Epigenetic histone methylation modulates fibrotic gene expression. J Am Soc Nephrol. 2010 Dec;21(12):2069-80.

90. Verrecchia F, Chu ML, Mauviel A. Identification of novel TGF-beta /Smad gene targets in dermal fibroblasts using a combined cDNA microarray/promoter transactivation approach. J Biol Chem. 2001 May 18;276(20):17058-62.

91. Chung AC, Zhang H, Kong YZ, et al. Advanced glycation end-products induce tubular CTGF via TGF-beta-independent Smad3 signaling. J Am Soc Nephrol. 2010 Feb;21(2):249-60.

92. Dennler S, Itoh S, Vivien D, ten Dijke P, Huet S, Gauthier JM. Direct binding of Smad3 and Smad4 to critical TGF beta-inducible elements in the promoter of human plasminogen activator inhibitor-type 1 gene. EMBO J. 1998 Jun 1;17(11):3091-100.

93. Yang F, Chung AC, Huang XR, Lan HY. Angiotensin II induces connective tissue growth factor and collagen I expression via transforming growth factor-betadependent and -independent Smad pathways: the role of Smad3. Hypertension. 2009 Oct;54(4):877-84.

94. Lan HY. Diverse roles of TGF-beta/Smads in renal fibrosis and inflammation. Int J Biol Sci. 2011;7(7):1056-67.

95. Fujimoto M, Maezawa Y, Yokote K, et al. Mice lacking Smad3 are protected against streptozotocin-induced diabetic glomerulopathy. Biochem Biophys Res Commun. 2003 Jun 13;305(4):1002-7.

96. Li JH, Huang XR, Zhu HJ, et al. Advanced glycation end products activate Smad signaling via TGF-beta-dependent and independent mechanisms: implications for diabetic renal and vascular disease. FASEB J. 2004 Jan;18(1):176-8.

97. Wang W, Huang XR, Canlas E, et al. Essential role of Smad3 in angiotensin II-induced vascular fibrosis. Circ Res. 2006 Apr 28;98(8):1032-9.

98. Martens JH, Verlaan M, Kalkhoven E, Zantema A. Cascade of distinct histone modifications during collagenase gene activation. Mol Cell Biol. 2003 Mar;23(5):180816. 
99. Pradhan S, Chin HG, Esteve PO, Jacobsen SE. SET7/9 mediated methylation of nonhistone proteins in mammalian cells. Epigenetics. 2009 Aug 16;4(6):383-7.

100. Dhayalan A, Kudithipudi S, Rathert P, Jeltsch A. Specificity analysis-based identification of new methylation targets of the SET7/9 protein lysine methyltransferase. Chem Biol. 2011 Jan 28;18(1):111-20.

101. Chuikov S, Kurash JK, Wilson JR, et al. Regulation of p53 activity through lysine methylation. Nature. 2004 Nov 18;432(7015):353-60.

102. Esteve PO, Chin HG, Benner J, et al. Regulation of DNMT1 stability through SET7mediated lysine methylation in mammalian cells. Proc Natl Acad Sci U S A. 2009 Mar 31;106(13):5076-81.

103. Subramanian K, Jia D, Kapoor-Vazirani P, et al. Regulation of estrogen receptor alpha by the SET7 lysine methyltransferase. Mol Cell. 2008 May 9;30(3):336-47.

104. Kouskouti A, Scheer E, Staub A, Tora L, Talianidis I. Gene-specific modulation of TAF10 function by SET9-mediated methylation. Mol Cell. 2004 Apr 23;14(2):175-82.

105. Yang J, Huang J, Dasgupta M, et al. Reversible methylation of promoter-bound STAT3 by histone-modifying enzymes. Proc Natl Acad Sci U S A. 2010 Dec 14;107(50):21499504.

106. Saraheimo M, Teppo AM, Forsblom C, Fagerudd J, Groop PH. Diabetic nephropathy is associated with low-grade inflammation in Type 1 diabetic patients. Diabetologia. 2003 Oct;46(10):1402-7.

107. Shikano M, Sobajima H, Yoshikawa H, et al. Usefulness of a highly sensitive urinary and serum IL-6 assay in patients with diabetic nephropathy. Nephron. 2000 May;85(1):81-5.

108. Tuttle HA, Davis-Gorman G, Goldman S, Copeland JG, McDonagh PF. Proinflammatory cytokines are increased in type 2 diabetic women with cardiovascular disease. J Diabetes Complications. 2004 Nov-Dec;18(6):343-51.

109. Mirza S, Hossain M, Mathews C, et al. Type 2-diabetes is associated with elevated levels of TNF-alpha, IL-6 and adiponectin and low levels of leptin in a population of Mexican Americans: a cross-sectional study. Cytokine. 2012 Jan;57(1):136-42.

110. Berthier CC, Zhang H, Schin M, et al. Enhanced expression of Janus kinase-signal transducer and activator of transcription pathway members in human diabetic nephropathy. Diabetes. 2009 Feb;58(2):469-77.

111. Simon AR, Rai U, Fanburg BL, Cochran BH. Activation of the JAK-STAT pathway by reactive oxygen species. Am J Physiol. 1998 Dec;275(6 Pt 1):C1640-52.

112. Huang JS, Guh JY, Chen HC, Hung WC, Lai YH, Chuang LY. Role of receptor for advanced glycation end-product (RAGE) and the JAK/STAT-signaling pathway in AGEinduced collagen production in NRK-49F cells. J Cell Biochem. 2001;81(1):102-13.

113. Lu TC, Wang ZH, Feng X, et al. Knockdown of Stat3 activity in vivo prevents diabetic glomerulopathy. Kidney Int. 2009 Jul;76(1):63-71.

114. Rawlings JS, Rosler KM, Harrison DA. The JAK/STAT signaling pathway. J Cell Sci. 2004 Mar 15;117(Pt 8):1281-3.

115. Yasukawa $\mathbf{H}$, Ohishi M, Mori $\mathbf{H}$, et al. IL-6 induces an anti-inflammatory response in the absence of SOCS3 in macrophages. Nat Immunol. 2003 Jun;4(6):551-6.

116. Ortiz-Munoz G, Lopez-Parra V, Lopez-Franco 0, et al. Suppressors of cytokine signaling abrogate diabetic nephropathy. J Am Soc Nephrol. 2010 May;21(5):763-72.

117. Gaughan L, Stockley J, Wang N, et al. Regulation of the androgen receptor by SET9mediated methylation. Nucleic Acids Res. 2011 Mar;39(4):1266-79.

118. O'Meara NM, Blackman JD, Ehrmann DA, et al. Defects in beta-cell function in functional ovarian hyperandrogenism. J Clin Endocrinol Metab. 1993 May;76(5):12417. 
119. Dunaif A, Finegood DT. Beta-cell dysfunction independent of obesity and glucose intolerance in the polycystic ovary syndrome. J Clin Endocrinol Metab. 1996 Mar;81(3):942-7.

120. Liu S, Navarro G, Mauvais-Jarvis F. Androgen excess produces systemic oxidative stress and predisposes to beta-cell failure in female mice. PLoS One. 2010;5(6):e11302.

121. Pirola L, Balcerczyk A, Tothill RW, et al. Genome-wide analysis distinguishes hyperglycemia regulated epigenetic signatures of primary vascular cells. Genome Res. 2011 Oct;21(10):1601-15.

122. Giacco F, Brownlee M. Oxidative stress and diabetic complications. Circ Res. 2010 Oct 29;107(9):1058-70.

123. Noyman I, Marikovsky M, Sasson S, et al. Hyperglycemia reduces nitric oxide synthase and glycogen synthase activity in endothelial cells. Nitric Oxide. 2002 Nov;7(3):187-93.

124. Zhang Q, Malik P, Pandey D, et al. Paradoxical activation of endothelial nitric oxide synthase by NADPH oxidase. Arterioscler Thromb Vasc Biol. 2008 Sep;28(9):1627-33.

125. Du X, Edelstein D, Obici S, Higham N, Zou MH, Brownlee M. Insulin resistance reduces arterial prostacyclin synthase and eNOS activities by increasing endothelial fatty acid oxidation. J Clin Invest. 2006 Apr;116(4):1071-80.

126. Shen X, Zheng S, Metreveli NS, Epstein PN. Protection of cardiac mitochondria by overexpression of MnSOD reduces diabetic cardiomyopathy. Diabetes. 2006 Mar;55(3):798-805.

127. Otero P, Bonet B, Herrera E, Rabano A. Development of atherosclerosis in the diabetic BALB/c mice. Prevention with Vitamin E administration. Atherosclerosis. 2005 Oct;182(2):259-65.

128. Zhang Y, Wada J, Hashimoto I, et al. Therapeutic approach for diabetic nephropathy using gene delivery of translocase of inner mitochondrial membrane 44 by reducing mitochondrial superoxide production. J Am Soc Nephrol. 2006 Apr;17(4):1090-101.

129. DeRubertis FR, Craven PA, Melhem MF. Acceleration of diabetic renal injury in the superoxide dismutase knockout mouse: effects of tempol. Metabolism. 2007 Sep;56(9):1256-64.

130. Kowluru RA, Kowluru V, Xiong Y, Ho YS. Overexpression of mitochondrial superoxide dismutase in mice protects the retina from diabetes-induced oxidative stress. Free Radic Biol Med. 2006 Oct 15;41(8):1191-6.

131. Vincent AM, Russell JW, Sullivan KA, et al. SOD2 protects neurons from injury in cell culture and animal models of diabetic neuropathy. Exp Neurol. 2007 Dec;208(2):21627.

132. Kurash JK, Lei H, Shen Q, et al. Methylation of p53 by Set7/9 mediates p53 acetylation and activity in vivo. Mol Cell. 2008 Feb 15;29(3):392-400. 